CDF/PUB/ELECTROWEAK/PUBLIC/7725

Version 1.1

June 30, 2005

Proceedings of DIS05

Madison, Wisconsin, April 27-May 1, 2005 


\title{
W Asymmetry and Z Rapidity Measurements
}

\author{
Y. S. Chung \\ Department of Physics and Astronomy, University of Rochester, Rochester, New York 14627-0171
}

\begin{abstract}
The measurements of $\mathrm{W}$ charge asymmetry and $\mathrm{Z}$ rapidity distributions are inputs to constrain the parton distribution functions at high $Q^{2}$. The $\mathrm{CDF}$ and $\mathrm{D} \emptyset$ experiments at the Tevatron have analyzed up to $340 \mathrm{pb}^{-1}$ of Run 2 data to measure the $\mathrm{W}$ charge asymmetry and $\mathrm{Z}$ rapidity. The measurements are in generally good agreement with predictions.
\end{abstract}

Keywords: asymmetry rapidity PDF

PACS: $12.38 . \mathrm{Qk}$

\section{INTRODUCTION}

The $\mathrm{W}$ and $\mathrm{Z}$ bosons are primarily produced by a quark and anti-quark annihilation in $p \bar{p}$ collisions at the Tevatron. Many precision measurements at the Tevatron are limited by the uncertainties on the parton distribution functions (PDFs). These include the $\mathrm{W}$ mass, cross section, transverse momentum distributions of $\mathrm{W}$ and $\mathrm{Z}$ bosons, and so on. For the $\mathrm{W}$ mass measurement in the CDF experiment, the current systematic uncertainty from PDF uncertainties is about $15 \mathrm{MeV} / c^{2}$ [1] and will become more significant as data accumulated.

The measurement of the $\mathrm{W}$ charge asymmetry provides input to the ratio of $u$ and $d$ quark components of PDFs, especially at the medium/high $x$. In the $p \bar{p} \rightarrow Z$ process, the momentum fraction of a parton is related to the $\mathrm{Z}$ rapidity $(y)^{1}$. Analysis of the high $y$ region probes the PDFs at both high and low momentum fraction.

\section{W ASYMMETRY MEASUREMENT}

The $W^{+}\left(W^{-}\right)$bosons at the Tevatron are produced primarily by the annihilation of $u(d)$ quarks in the proton and $\bar{d}(\bar{u})$ quarks in the anti-proton. Because u quarks carry, on average, more momentum than $\mathrm{d}$ quarks, the $W^{+}$tend to follow the direction of the incoming proton and the $W^{-}$that of the anti-proton.

The $W^{ \pm}$charge asymmetry

$$
A\left(y_{W}\right)=\frac{d \sigma_{+} / d y_{W}-d \sigma_{-} / d y_{W}}{d \sigma_{+} / d y_{W}+d \sigma_{-} / d y_{W}},
$$

where the subscript $(+,-)$ denotes the charge of the $W$ and $y_{W}$ is the rapidity of the $W$ bosons is a sensitive probe of the momentum fraction difference between $u$ and $d$ quarks

$1 y=\frac{1}{2} \ln \frac{\left(E+P_{z}\right)}{\left(E-P_{z}\right)}$ 
in the $Q^{2} \approx M_{W}^{2}$ region. A precise measurement of the $W$ asymmetry serves as a valuable constraint on the $u$ and $d$ quark momentum distributions. For $p \bar{p}$ collisions in leadingorder parton model, $A\left(y_{W}\right)$ is given approximately by

$$
A\left(y_{W}\right) \approx \frac{u\left(x_{1}\right) d\left(x_{2}\right)-d\left(x_{1}\right) u\left(x_{2}\right)}{u\left(x_{1}\right) d\left(x_{2}\right)+d\left(x_{1}\right) u\left(x_{2}\right)},
$$

where $x_{1,2}=x_{0} e^{ \pm y_{W}}, x_{0}=M_{W} / \sqrt{s}$, and $s$ is the center of mass energy. The asymmetry, $A\left(y_{W}\right)$, is related to the slope of $\mathrm{d} / \mathrm{u}$ at $Q^{2}=M_{W}^{2}$ and in the moderate $x$ region of W production.

The rapidity of the $\mathrm{W}$ boson is not measured because the longitudinal momentum of the neutrino can not be experimentally determined. Therefore, we measure the lepton asymmetry, which is a convolution of the $\mathrm{W}$ production charge asymmetry and the V-A couplings in the W decay. The lepton asymmetry is still sensitive to PDFs and is defined as:

$$
A\left(y_{l}\right)=\frac{d \sigma_{+} / d y_{l}-d \sigma_{-} / d y_{l}}{d \sigma_{+} / d y_{l}+d \sigma_{-} / d y_{l}} .
$$

The CDF experiment measured the forward-backward charge asymmetry of electrons from $\mathrm{W}$ boson decays in $p \bar{p}$ collisions at $\sqrt{s}=1.96 \mathrm{TeV}$ using a data sample of 170 $p b^{-1}$ of Run 2 data [2]. Candidate $\mathrm{W} \rightarrow e v$ events were required to have exactly one electron candidate of $E_{T}>25 \mathrm{GeV}$, with $\not_{T}>25 \mathrm{GeV}$ and transverse mass in the range $50 \mathrm{GeV} / c^{2}<M_{T}<100 \mathrm{GeV} / c^{2}$.

The CDF experiment improved its sensitivity to the PDFs by reducing the decay asymmetry. The measurement separates the asymmetry measurement into two bins: 25 $\mathrm{GeV}<E_{T}<35 \mathrm{GeV}$ and $35 \mathrm{GeV}<E_{T}<45 \mathrm{GeV}$ (Figure 1 and 2). This is done for the first time and the separation into different bins is a novel addition to the previous measurements. Predictions from CTEQ [3] and MRST [4] PDFs using a NLO RESBOS calculation [5] are shown for comparison.

The CDF Collaboration is additionally developing a new method to measure the $\mathrm{W}$ asymmetry directly by partially reconstructing $\mathrm{W}$ rapidity using a $\mathrm{W}$ mass.

\section{Z RAPIDITY DISTRIBUTION}

Measurement of the rapidity distribution of Drell-Yan pairs in the $\mathrm{Z}$ boson mass region provides a stringent test of PDFs. At leading order (LO), the momentum fraction, $x_{+}\left(x_{-}\right)$, carried by the parton from the proton (anti-proton) is related to the rapidity of the $\mathrm{Z}$ boson via the equation:

$$
x_{ \pm}=\frac{M_{Z}}{\sqrt{s}} e^{ \pm y}
$$

where $M_{Z}$ is the mass of $Z$ boson. Therefore the analysis of the high $y$ region probes the high $x$ as well as low $x$ region.

The $\mathrm{D} \emptyset$ experiment measures the rapidity distribution, $d \sigma / d y$, of the Drell-Yan process in the dielectron's mass range between 71 and $111 \mathrm{GeV} / c^{2}$ [6]. The data were collected with the $\mathrm{D} \emptyset$ detector in Tevatron $p \bar{p}$ collisions at $\sqrt{s}=1.96 \mathrm{TeV}$ using a data 


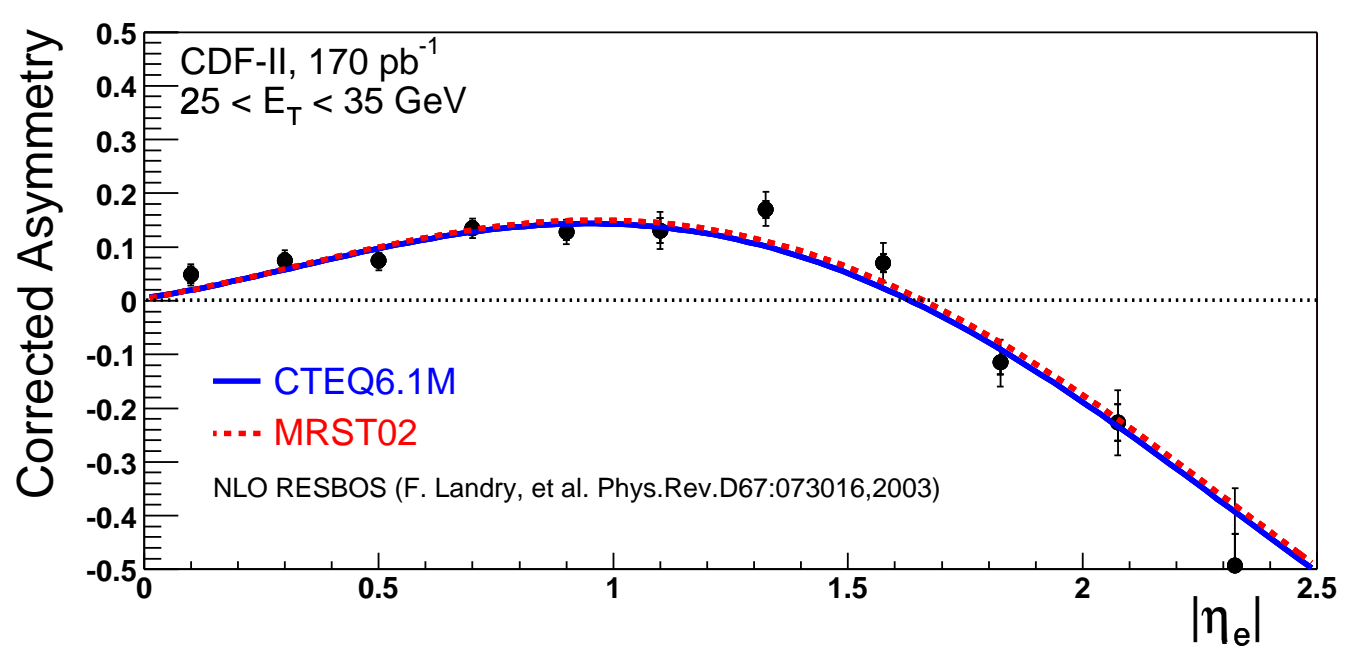

FIGURE 1. The measured lepton charge asymmetry for $25 \mathrm{GeV}<E_{T}<35 \mathrm{GeV}$ with predictions from CTEQ6.1M (solid) and MRST02 (dashed) PDFs using a NLO RESBOS calculation. Both statistical and total (statistical+systematic) uncertainties are shown.

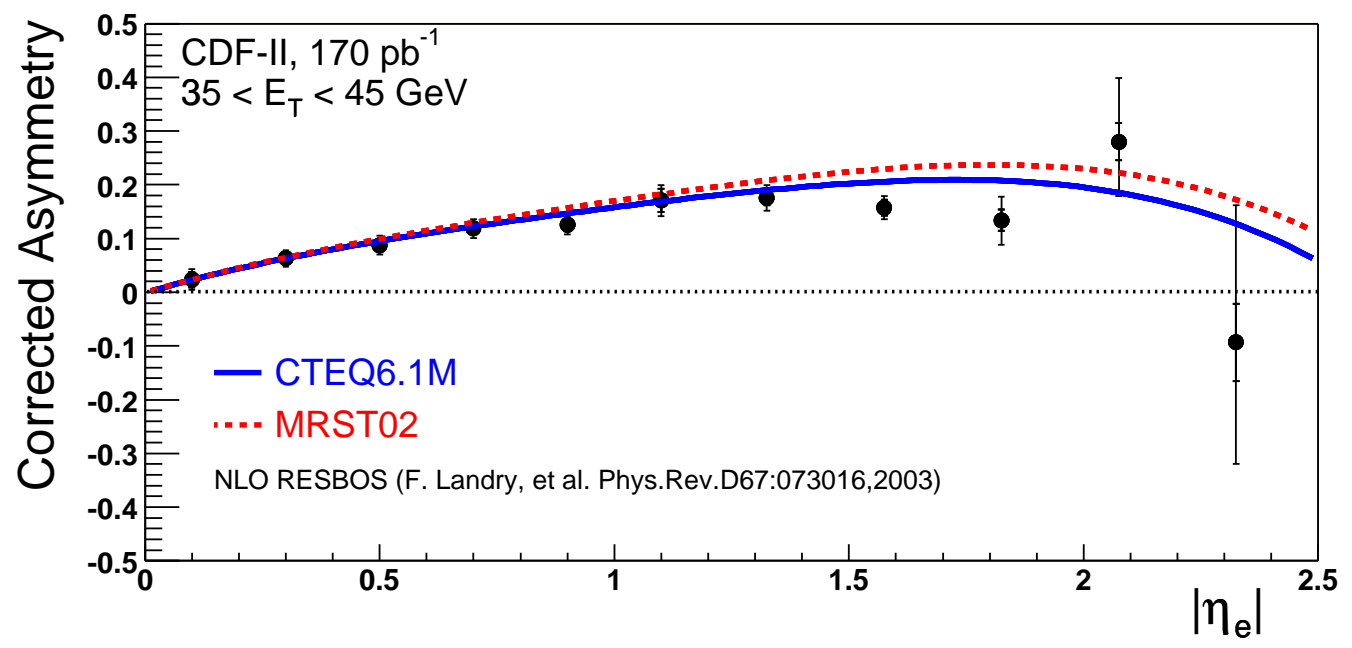

FIGURE 2. The measured lepton charge asymmetry for $35 \mathrm{GeV}<E_{T}<45 \mathrm{GeV}$ with predictions from CTEQ6.1M (solid) and MRST02 (dashed) PDFs using a NLO RESBOS calculation. Both statistical and total (statistical+systematic) uncertainties are shown.

sample of $337 p b^{-1}$ of Run 2 data. The final result, with statistical and systematic errors, is shown in Figure 3. The measurement of $d \sigma / d y$ is compared with a prediction using an NNLO calculation based on the MRST 2001 PDFs [7]. The D $\varnothing$ forward calorimeters provide data over almost the entire rapidity range accessible at Tevatron. Measurements are in generally good agreement with the prediction. 


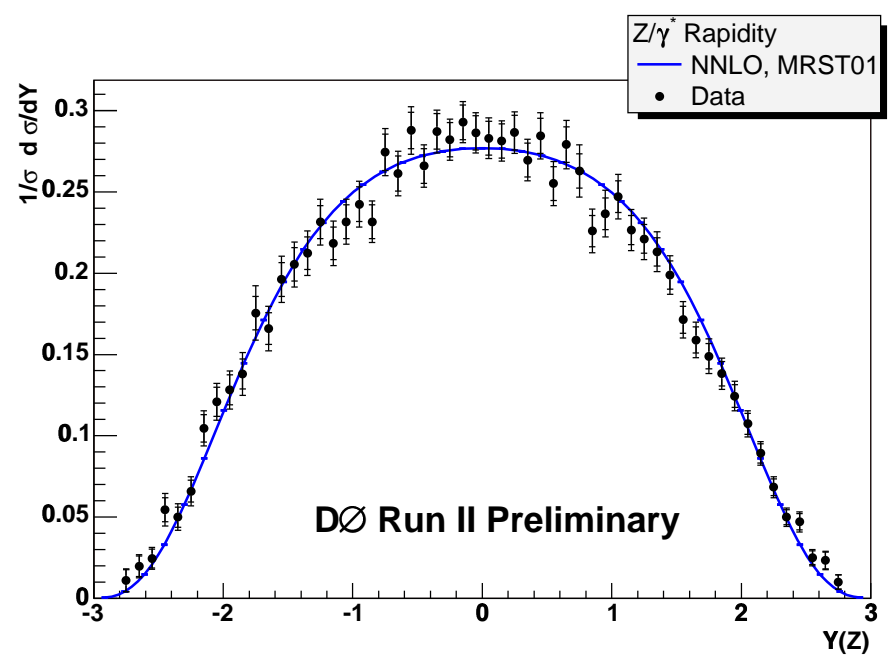

FIGURE 3. The $\mathrm{D} \varnothing$ measurement of the $\mathrm{Z}$ rapidity distribution. The outer error bars show the total error (statistical+systematic), while the inner error bars indicate the statistical error alone. The solid line shows the NNLO prediction based on the MRST 2001 PDFs.

\section{CONCLUSIONS}

The CDF experiment have measured the W charge asymmetry. Since the previous measurements upon which the current predictions are based are least constraining for $|\eta|>1$ and do not separate the $E_{T}$ dependence, inclusion of the new $\mathrm{W}$ asymmetry results will further constrain future fits and improve the predictions. The $\mathrm{D} \varnothing$ measurement of the $\mathrm{Z}$ rapidity distribution is in generally good agreement with theory prediction. We expect that Tevatron $\mathrm{Z}$ and $\mathrm{W}$ measurements will provide important input to constrain the PDFs, especially in the high $x$ region.

\section{ACKNOWLEDGMENTS}

I would like to thank the workshop organizers and working group leaders for their invitation and a wonderful workshop. Also many thanks to the electroweak conveners of both the CDF and DØ Collaborations for providing material and valuable comments.

\section{REFERENCES}

1. CDF 2 Collaboration, CDF Run 2 W mass, hep-ex/0505064 (2005), hep-ex/0506016 (2005).

2. CDF 2 Collaboration, D. Acosta et al, Phys. Rev. D 71, 051104(R) (2005).

3. J. Pumplin, D. R. Stump, J. Huston, H. L. Lai, P. Nadolsky, and W. K. Tung, JHEP 0207012 (2002).

4. A. Martin, R. Roberts, W. Stirling, and R. Thorne, Eur. Phys. J. C 4, 463 (1998).

5. C. Balazs and C.P. Yuan, Phys. Rev. D, 56, 5558 (1997).

6. The DØ Collaboration, http://www-d0.fnal.gov/Run2Physics/WWW/results/ew.htm.

7. C. Anastasiou, L. Dixon, K. Melnikov and F. Petriello, Phys. Rev. D 69, 094008 (2004). 